\title{
Appendicitis Causing Ascending Infection Through Ventriculoperitoneal Shunt
}

Tetsuro Kawazoe ${ }^{1}$, Masahiko Sugiyama ${ }^{1}$, Kimiaki Hashiguchi ${ }^{2}$, Ryota Nakanishi ${ }^{1}$, Junji Kurashige ${ }^{1}$, Yuichiro Nakashima $^{1}$, Hiroshi Saeki ${ }^{1}$, Eiji Oki ${ }^{1}$, Yoshihiko Maehara ${ }^{1}$

Departments of ${ }^{1}$ Surgery and Science $\&{ }^{2}$ Neurosurgery, Graduate School of Medical Sciences, Kyushu University, Fukuoka, Japan.

\section{Corresponding Author:}

Dr. Eiji Oki

Email: okieiji@surg2.med.kyushu-u.ac.jp

This is an Open Access article distributed under the terms of the Creative Commons Attribution License (creativecommons.org/ licenses/by/3.0).

Received Accepted

October 5, 2017

Published

August 7, 2018

September 15, 2018

\begin{abstract}
Background: Ventriculo-peritoneal shunts (VPS) are devices that treat neurologic disorders such as hydrocephalus. In patients with VPS, it is occasionally difficult to diagnose appendicitis and to determine the best treatment plan. Case Report: A 17-yearold male with a VPS implanted during childhood complained of right lower quadrant abdominal pain. Laboratory data was consistent with systemic inflammation and computed tomography (CT) findings demonstrated appendicitis with catarrahalis. Based on the low severity of infection and the patient's desire not to undergo surgery, he was conservatively treated with antibiotics. Unfortunately, he developed three subsequent episodes of acute appendicitis. During the third episode, in the absence of appendix perforation, ascending infection through the VPS caused meningitis. He thus underwent VPS removal and appendectomy. Conclusion: Appendicitis in a patient with VPS should be treated surgically as soon as possible, even in patients with a mild, non-perforated infection.
\end{abstract}

Keywords: Appendectomy, Appendicitis, Hydrocephalus, Meningitis, Ventriculoperitoneal Shunt.

\section{Introduction}

Ventriculo-peritoneal shunts (VPS) are surgical devices that are placed between the brain ventricle and the peritoneal space for the purpose of draining excess cerebrospinal fluid from the ventricular system. VPS are one of the best treatment modalities for neurologic disorders such as hydrocephalus. VPS are widely used; each year approximately 16,000 patients required VPS implantation in Japan [1]. However, the cumulative complication rate of VPS at 5 years is estimated at 32\% [2]. Many of these complications are associated with abdominal pathologies that result in shunt malfunction or infection. Although there is some literature detailing the complications of VPS [3] and, in particular, the complications of VPS after abdominal surgery [4], there is no consensus regarding the treatment of abdominal infections such as appendicitis in patients with VPS. Furthermore, there are no guidelines for the peri-operative management of VPS, even with the risk of ascending infection. In this report, we present a patient with VPS who developed meningitis secondary to unperforated appendicitis.

\section{Case Report}

A 17-year-old Japanese male was admitted to our hospital with sharp pain in the right lower quadrant of the abdomen. His past medical history was significant for sub-ependymal hemorrhage in his prenatal period, and he had a VPS implanted for secondary hydrocephalus at six months of age.

His vital signs were within the normal range, but physical exam revealed tenderness at McBurney's point without evident muscular guarding. Laboratory data was consistent with systemic inflammation, with elevation of white blood cells (WBC, 9340/ $\mu \mathrm{L}$ ) and C-reactive 
protein (CRP, $0.83 \mathrm{mg} / \mathrm{dL}$ ). He did not complain of headache, and his cerebrospinal fluid (CSF) examination revealed no evidence of meningitis [no WBC, normal total protein (TP), glucose, and lactate dehydrogenase (LDH)]. Abdominal X-ray showed the VPS which was implanted in his childhood [Fig.1]. Abdominal computed tomography (CT) showed an appendiceal fecalith, which was approximately $5 \mathrm{~mm}$ in diameter; the appendix was slightly swollen, with a wall thickness of $8 \mathrm{~mm}$ in diameter. The distal end of the VPS was located in the right lower quadrant of the abdomen [Fig.2]. Based on these findings, he was diagnosed with acute appendicitis, without ascending infection of the VPS.

Urgent appendectomy was recommended, but the patient and his family opted for conservative treatment. He was thus treated with intravenous antibiotics. His symptoms and inflammatory findings improved, and he was discharged on hospital day six. On the $39^{\text {th }}$ day after the first hospitalization, he re-presented to our hospital with recurrent abdominal pain. He again had no meningeal symptoms. He was diagnosed with relapsed appendicitis. Appendectomy was recommended for relapsed appendicitis, but he and his family again opted for conservative management. After treatment with intravenous antibiotics, his inflammatory findings improved, and he was discharged on the $49^{\text {th }}$ day after the first hospitalization.

On the $54^{\text {th }}$ day after the first hospitalization, however, he came to our hospital again with abdominal pain. At that time, his body temperature was elevated to $38.0^{\circ} \mathrm{C}$, and his physical examination findings included headache, nausea, and right lower quadrant abdominal pain. Laboratory findings showed an elevated WBC

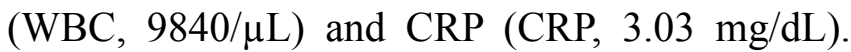
Moreover, his CSF findings revealed an elevation of the WBC (WBC, 9588/ $\mu \mathrm{L}$ ), TP (TP, $261 \mathrm{mg} /$ $\mathrm{dl})$, and $\mathrm{LDH}(\mathrm{LDH}, 80 \mathrm{U} / \mathrm{L})$. He was diagnosed with recurrent appendicitis with meningitis due

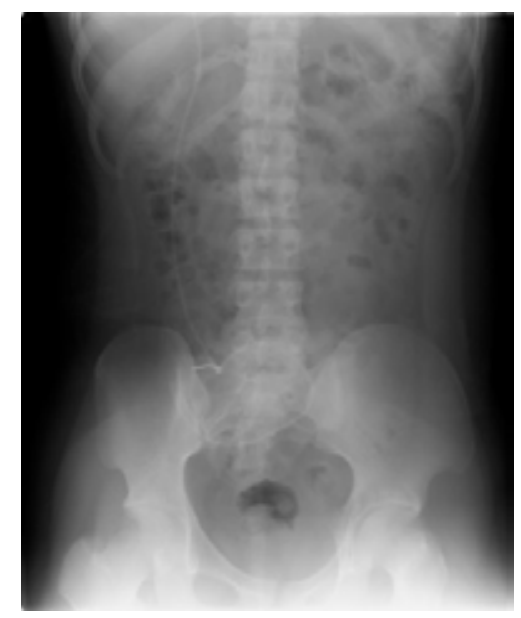

Fig.1: Abdominal X-ray showed a ventriculo-peritoneal shunt in the right side of the abdomen.

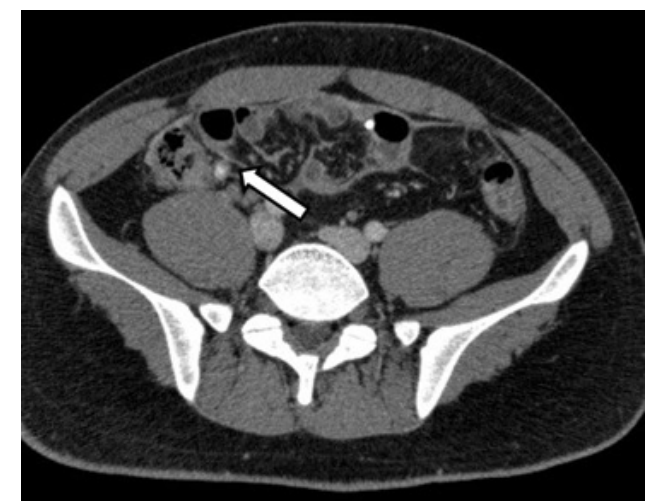

Fig.2: A fecalith, approximately $5 \mathrm{~mm}$ in diameter, was found in the appendix (white arrow). The appendix swelled to $8 \mathrm{~mm}$ in diameter.

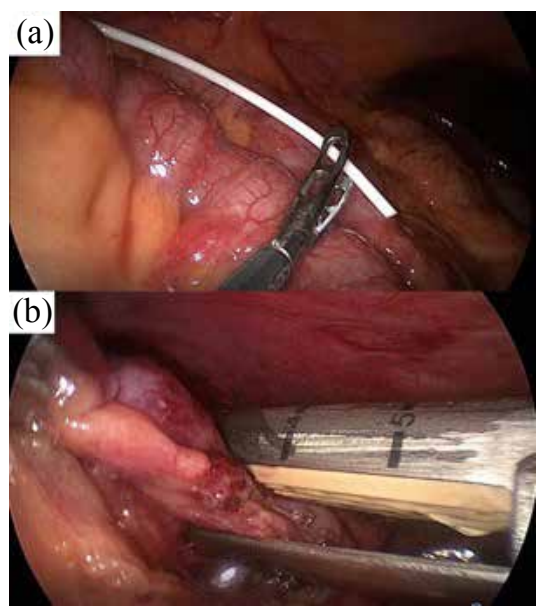

Fig.3(a): The distal end of the ventriculoperitoneal shunt was observed in the patient's abdomen near the appendix. (b): The appendix was red and swollen. It was cut with an autosuture. 
to ascending infection of the VPS, and the patient and his family agreed to surgical intervention. During the first surgical stage, the VPS was ligated distal to the VPS valve, and then cut behind the right auricle. During the second surgical stage, the patient underwent laparoscopic appendectomy. The appendix was noted to be swollen and attached to the abdominal wall by adhesions, and small amount of exudative ascites was observed [Fig.3]. After the appendectomy, a spinal drain was inserted and externalized for decompression of the medullary cavity. Propionibacterium acnes was isolated from both the spinal fluid and the distal end of the VPS.

On post-operative day 7 (61 days after the first hospitalization), the spinal drain was removed and his ventricle gradually dilated over time. However, his headache improved and the ventricular dilation appeared to stabilize. He was discharged 34 days after the operation (88 days after the first hospitalization). However, he soon re-presented with a worsening headache. Ventricular dilation was recognized on head CT, and VPS reinsertion was performed on post-operative day 49 (103 days after the first hospitalization). After 8 months of post-appendectomy follow-up, he had no further complications, with the exception of mild headaches.

\section{Discussion}

VPS has been widely used to treat patients with hydrocephalus. The peritoneum is well suited for
CSF absorption. Ventriculo-atrial shunt systems, preferred in the past, have been associated with a wide range of complications such as obstruction, infection, endocarditis and thrombo-emboli. VPS systems have a lower incidence of complications. In Japan, the use of CSF shunting has been increasing; currently, 16,000 cerebrospinal shunting operations are performed each year [1]. But a number of complications, such as proximal, valvular, and distal obstruction and infection, have been associated with the use of VPS [5]. Such complications have been reported in two large case series with a frequency upto 24\% [6,7]. Among these complications, shunt infection, ascending central nervous system (CNS) infection, and meningitis required immediate attention.

The diagnosis of a child with a VPS who has an acute abdomen can be difficult [8] because of the large differential diagnoses, which includes appendicitis. Furthermore, when a patient with VPS undergoes abdominal surgery, the surgeon must determine the appropriate peri-operative VPS management. Several previous reports have described appendicitis in patients with VPS [Table 1] [4,9-11]. In these reports, all 22 patients were treated surgically with appendectomy, rather than conservatively with antibiotics. The reports included 12 cases of perforated appendicitis, and six of these patients underwent VPS externalization at the time of appendectomy. Four of the six patients who did not undergo VPS externalization

Table 1

\begin{tabular}{|c|c|c|c|c|c|}
\hline Author & $\begin{array}{l}\text { Number } \\
\text { of cases }\end{array}$ & Severity & Operation & $\begin{array}{l}\text { Externalization } \\
\text { of VPS during } \\
\text { appendectomy }\end{array}$ & Ascending infection \\
\hline Ein et al. [11] & 8 & 3 perforated cases & 8 & 3 (ruptured cases) & 0 \\
\hline $\begin{array}{l}\text { Mortellaro } \\
\text { et al. [5] }\end{array}$ & 3 & $\begin{array}{l}2 \text { perforated or } \\
\text { gangrenous cases }\end{array}$ & 3 & 0 & 2 ( 1 case required shunt externalization) \\
\hline
\end{tabular}


suffered from VPS complications, including three ascending infections and one CSF pseudocyst. Three of these patients subsequently underwent VPS externalization. There were no cases of ascending infection in the absence of perforated or gangrenous appendicitis.

Based on these prior reports, the major treatment policy for acute appendicitis in the presence of a VPS might be to remove the appendix and leave the shunt in place [10]. Li et al. concluded that there is minimal risk for VPS malfunction or infection among patients undergoing clean and clean-contaminated abdominal surgeries [3]. In the previous reports, VPS externalization was generally required in patients with perforated appendicitis. Six of 12 patients underwent VPS externalization at the time of appendectomy. There is no evidence to suggest that clamping or externalization of the catheter is necessary in non-perforated appendicitis [12]. However, previous studies have reported that the shunt requires externalization in the setting of perforated appendicitis [3,13]. Early surgical intervention may prevent ascending infection from non-perforated appendicitis to the CSF. In our case, definite perforation of the appendix was not observed, but the patient developed chronic appendicitis, which resulted in ascending infection of the CSF during the prolonged treatment course. Hence, even patients with non-perforated chronic appendicitis may develop ascending infection to the CSF. Early diagnosis and surgical treatment of appendicitis is important in patients with VPS.

\section{Conclusion}

This case demonstrated that conservative treatment of chronic appendicitis resulted in ascending CNS infection and meningitis in a patient with VPS. Hence, conservative treatment of appendicitis should not be recommended in a patient with VPS.

Contributors: KT and MS conceived of this case presentation and drafted the manuscript. KH, RN, JK, YN, HS provided critical inputs into the manuscript and participated in the treatment of this patient. EO and YM revised the article. EO will act as guarantor. All authors read and approved the final manuscript.

Funding: None; Competing interests: None stated.

\section{References}

1. Kamei M, Kikuchi N, Ichimura H, Chujo M, Takahashi Y, Sugio K. A case of breast cancer involving a ventriculoperitoneal shunt. Surg Case Rep. 2016;2:8.

2. Wu Y, Green NL, Wrensch MR, Zhao S, Gupta N. Ventriculoperitoneal shunt complications in California: 1990 to 2000. Neurosurgery 2007;61:557-562; discussion. 562-553.

3. Li G, Dutta S. Perioperative management of ventriculoperitoneal shunts during abdominal surgery. Surg Neurol. 2008;70:492-495; discussion 495-497.

4. Mortellaro VE, Chen MK, Pincus D, Kays DW, Islam $\mathrm{S}$, Beierle EA. Infectious risk to ventriculo-peritoneal shunts from gastrointestinal surgery in the pediatric population. J Pediatr Surg. 2009;44:1201-1205.

5. Blount JP, Campbell JA, Haines SJ. Complications in ventricular cerebrospinal fluid shunting. Neurosurg Clin N Am. 1993;4:633-656.

6. Grosfeld JL, Cooney DR, Smith J, Campbell RL. Intraabdominal complications following ventriculoperitoneal shunt procedures. Pediatrics. 1974;54:791-796.

7. Patrick D, Marcotte P, Garber GE. Acute abdomen in the patient with a ventriculoperitoneal shunt. Can J Surg. 1990;33:37-40.

8. Rush DS, Walsh JW, Belin RP, Pulito AR. Ventricular sepsis and abdominally related complications in children with cerebrospinal fluid shunts. Surgery. 1985;97:420427.

9. Pumberger W, Lobl M, Geissler W. Appendicitis in children with a ventriculoperitoneal shunt. Pediatr Neurosurg. 1998;28:21-26.

10. Ein SH, Miller S, Rutka JT. Appendicitis in the child with a ventriculo-peritoneal shunt: a 30-year review. J Pediatr Surg. 2006;41:1255-1258.

11. Barina AR, Virgo KS, Mushi E, Bahadursingh AM, Johnson FE. Appendectomy for appendicitis in patients with a prior ventriculoperitoneal shunt. The Journal of Surgical Research. 2007;141:40-44.

12. Hammill CW, Au T, Wong LL. Laparoscopic cholecystectomy in a patient with a ventriculoperitoneal shunt. Hawaii Medical Journal. 2010;69:103-104.

13. Rekate HL, Yonas H, White RJ, Nulsen FE. The acute abdomen in patients with ventriculoperitoneal shunts. Surg Neurol. 1979;11:442-445. 